\title{
Long-term Recording of LTP in Cultured Hippocampal Slices
}

\author{
Ken Shimono ${ }^{1,2}$, Michel Baudry ${ }^{3}$, Lam Ho $^{1}$, Makoto Taketani ${ }^{1,2}$ and Gary Lynch ${ }^{3}$ \\ ${ }^{1}$ Tensor Biosciences, Irvine, CA 92612; ${ }^{2}$ Panasonic Technology Company, 6550 Katella \\ Ave., Cypress, CA 90630; ${ }^{3}$ Dept. Psychiatry and Human Behavior, University of California, \\ 101 Theory Dr., suite 250, Irvine CA 92697; USA
}

\begin{abstract}
Long-term potentiation (LTP) was elicited by high frequency stimulation in hippocampal slices cultured on multi-electrode arrays. LTP lasting more than $1 \mathrm{~h}$ was recorded in $75 \%$ of slices, and a significant number of slices exhibited a non-decaying LTP that lasted more than $48 \mathrm{~h}$. LTP induction was completely and reversibly blocked by an antagonist of the NMDA receptor, APV. Our results suggest the possibility of using chronic recording in hippocampal slices cultured on multi-electrode arrays to study the mechanisms underlying LTP maintenance and stabilization.
\end{abstract}

\section{KEYWORDS}

LTP, hippocampus, organotypic culture, multielectrode array, NMDA, APV

\section{INTRODUCTION}

Long-term potentiation (LTP) is a long lasting increase in synaptic efficacy elicited by brief trains of high frequency activity. LTP has been observed in several cortical and subcortical pathways in

Reprint requests to: Ken Shimono, Tensor Biosciences, 101 Theory Dr., suite 250, Irvine CA 92612 USA, e-mail: kshimono@tensorbio.com in vivo and in vitro preparations. Because of its features of rapid induction, long duration, selectivity, and predominance in hippocampal pathways, LTP has been proposed to be a cellular mechanism for certain forms of information storage in the adult central nervous system (CNS) (Malenka \& Nicoll, 1999). Although LTP has been shown to persist for several weeks in vivo (Staubli \& Lynch, 1987), the question of its duration in in vitro preparations has rarely been addressed because of the difficulties of recording from slices for long periods. Few studies have used cultured hippocampal slices to evaluate mechanisms of LTP induction, expression, and maintenance. In general, LTP was found to exhibit similar features in cultured slices and in acute slices, with an increased probability of eliciting LTP and an increased amplitude of LTP with age in culture (Muller et al., 1993). No report has yet emerged, however, about LTP duration in cultured hippocampal slices.

Different types of mechanisms have been proposed to account for the increased synaptic efficacy at various stages of LTP expression in hippocampal field CA1 (for review, see Luscher et al., 2000). An initial activation of calcium/ calmodulin-dependent protein kinase has been suggested to increase single channel conductance (Benke et al., 1998) or to modify the distribution/ number of AMPA receptors (Lynch \& Baudry, 1991; Shi et al., 1999) or both. In later stages of LTP maintenance, the size of the postsynaptic density has been shown to increase (Desmond \& 
Levy, 1986), and new spines might form in the vicinity of activated spines (Lee et al., 1981; Chang \& Greenough, 1984; Geinisman et al., 1991; Toni et al., 1999). Although numerous studies have investigated mechanisms underlying LTP induction with a variety of techniques (for a review, see Malinow \& Malenka, 2002), most studies directed at understanding the mechanisms of LTP maintenance were carried out using morphological methods (for review, see Luscher et al., 2000), due to the difficulties inherent to the long-time recording of electrical signals with conventional electrophysiological methods using in vitro preparations. Nevertheless, several authors have proposed the existence of different cellular mechanisms for early and late stages of LTP, with the latter requiring protein synthesis. Developing a reliable electrophysiological method for the longterm monitoring of synaptic efficacy would therefore be very important to further understand the nature and properties of the mechanisms underlying the long-term maintenance and stabilization of LTP in an in vitro preparation.

In the present study, we have demonstrated the feasibility of the long-term monitoring of potentiated responses in cultured slices from rat hippocampus, using a recently reported technique of culturing hippocampal slices, obtained from postnatal day 11 rats, directly on multi-electrode arrays (MED probes) (Shimono et al., 2002). The potentiated responses recorded in CAl were maintained without significant decay for more than 2 days after tetanus stimulation of the Schaffer collaterals. This form of LTP was reversibly blocked by the NMDA receptor antagonist, APV, indicating that it involves the same mechanism of induction as LTP elicited by traditional approaches in acute hippocampal slices. This finding indicates that this approach for studying LTP can provide new insights into the mechanisms involved in LTP maintenance and stabilization.

\section{EXPERIMENTAL}

\section{Preparation of cultured hippocampal slices on MED probes}

The MED probes (Panasonic; MED-P515AP, each electrode: 50 × $50 \mu \mathrm{m}$, interpolar distance: $150 \mu \mathrm{m})$ were used as culturing dishes for hippocampal slices and for measuring field excitatory post-synaptic potentials (fEPSPs) elicited by the stimulation of various electrodes. Hippocampal slices were placed on MED probes in such a way that only field CA1 was covered by the whole electrode array. The detailed procedures for culturing hippocampal slices on MED probes are described in Shimono et al. (2002). Briefly, all procedures for culture preparation were carried out under a sterilized bench. Sprague-Dawley rats (11 days old) were sterilized with $70 \%$ ethanol, anesthetized and then sacrificed by decapitation, and the whole brain was removed. The brains were immediately soaked in sterile ice-cold MEM (pH 7.2; GIBCO; 61100), supplemented with HEPES $(25 \mathrm{mM})$, Tris $(10 \mathrm{mM})$, glucose $(10 \mathrm{mM})$, and $\mathrm{MgCl} 2(3 \mathrm{mM})$. Appropriate portions of the brain were trimmed by hand, and the remaining brain block was placed on the ice-cold stage of a vibrating tissue slicer (Leica; VT1000S). The thickness of the slices was set at $200 \mu \mathrm{m}$. The slices were gently taken off from the blade with a pipette. Each slice was trimmed, placed on the center of the MED probe coated with polyethylenimine, and positioned to cover the $8 \times 8$ micro-electrode array. After the section was positioned on the MED probe, the cutting solution was removed and the culture medium (2:1 mixture of Basal Medium Eagle [Sigma; B9638] and Earle Balanced Salts Solution [Sigma; E7510] supplemented with $20 \mathrm{mM}$

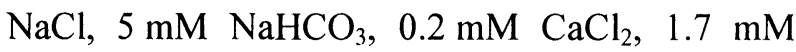
$\mathrm{MgSO}_{4}, 48 \mathrm{mM}$ glucose, $26.7 \mathrm{mM}$ HEPES, 5\% horse serum (GIBCO; 26050), and $10 \mathrm{ml} / \mathrm{L}$ penicillin 
streptomycin (GIBCO; 10378), adjusted to $\mathrm{pH} 7.2$ ) was added to the slice up to an interface level (approximately $250 \mu \mathrm{L}$ ). Sterile distilled water was added around the probe to increase humidity and to prevent over-drying of the culture medium in the MED probe. The slices on the MED probes were stored in a $\mathrm{CO}_{2}$ incubator at $34^{\circ} \mathrm{C}$. The medium was exchanged with half volume every day. The slices were cultured for about 10 days before initiating electrophysiological recordings.

\section{Electrophysiological recording and data analysis}

For electrophysiological recordings, the MED probes containing the slices were removed from the incubator and placed in a small $\mathrm{CO}_{2}$ incubator at $34{ }^{\circ} \mathrm{C}$ and connected to the stimulation/recording component of the multi-channel recording system (Panasonic: MED64 system). The medium was replaced with oxygenated sterile ACSF with $10 \mathrm{mM}$ HEPES. Evoked field potentials at all 64 sites were simultaneously recorded with the MED64 system at a $20 \mathrm{kHz}$ sampling rate. One of the planar microelectrodes of the 64 available was used for cathode of stimulation. Bipolar constant current pulses $(0.1 \mathrm{msec}$ duration) were delivered at $30 \%$ to $50 \%$ intensity of the current that produced the maximum fEPSP on each pathway. Stability was established routinely using MED64 Performer $^{\mathrm{TM}}$ software (Panasonic) for at least 15 min before the pre-tetanus stimulation baseline recording. Two independent sets of inputs converging on the same target set of pyramidal cells in CA1 were selected (independence of the pathways was tested by ensuring that no interaction occurred between pathways using crossed paired-pulse stimulation), and the responses were collected with alternative stimulation every $30 \mathrm{sec}$. Cultured slices that did not meet the above criteria were discarded. For LTP induction, three stimulus trains of 100 pulses (at $100 \mathrm{~Hz}$ ) with 10 -min inter-train intervals were applied as described by Frey and Morris (1997); low-frequency stimulation was continued for at least $1 \mathrm{~h}$ after the last high frequency train. After each recording session, ACSF was replaced with culture medium and the slices in the probes were returned to the $\mathrm{CO}_{2}$ incubator. A similar lowfrequency stimulation/recording procedure was repeated after 1 day and 2 days on those slices that exhibited LTP. The initial slopes and amplitudes of fEPSPs were determined and expressed as percentage of the average values measured during the 15 minutes baseline recording period. As similar results were obtained whether initial slopes or amplitudes of the responses were analyzed, only the amplitude data are shown in the figures. No attempt was made to measure population spike amplitudes as these are more difficult to reliably observe under our conditions. All data are presented as mean \pm S.E.M. One-way analysis of variance (ANOVA) was used for statistical analysis.

\section{RESULTS}

\section{Long-term recording in cultured hippocampal slices}

We previously demonstrated that culturing hippocampal slices directly on MED probes results in a tight adhesion of the slices on the probes, thus providing for stable maintenance of the stimulating and recording sites and thereby long-term extracellular recording in hippocampal field CA1 (Shimono et al, 2002).

Long-term potentiation was induced by delivering trains of high frequency to one of two inputs converging on one set of pyramidal cells in CA1, with the other input serving as control (Fig. 1). The fEPSPs evoked by stimulation on either test or control pathway were recorded before and 
$1 \mathrm{~h}, 1 \mathrm{~d}$, and $2 \mathrm{~d}$ after delivering tetanus stimulations to the test pathway.

Whereas the fEPSPs evoked in the control pathway remained stable during recording in the test pathway, potentiation of the evoked fEPSPs began immediately after tetanus and, in a subset of slices, lasted for more than $2 \mathrm{~d}$ without any significant decay over this period (Fig. 1B). Potentiation lasting more than $1 \mathrm{~h}$ occurred in 27 of 38 slices tested, and the number of slices in which LTP lasted more than $1 \mathrm{~d}$ was 15 of 27 slices. The data obtained from the long-term recordings of LTP that lasted more than $2 \mathrm{~d}(\mathrm{n}=8)$ are summarized in Fig. 1C. Field EPSP amplitudes in test and control pathways represented $143.2 \pm 3.2 \%$ and $110 \pm 2.2 \%$ of their respective pre-tetanus levels after $1 \mathrm{~h}, 155.5 \pm 2.5 \%$ and $113.4 \pm 2.4 \%$ after $1 \mathrm{~d}$, and $149.4 \pm 2.6 \%$ and $104.9 \pm 4.2 \%$ after $2 \mathrm{~d}$. The differences between the responses recorded in the test and control pathways at $1 \mathrm{~h}, 1 \mathrm{~d}$, and $2 \mathrm{~d}$ after tetanus were highly significant $(\mathrm{P}<0.0001, \mathrm{n}=8)$.
A

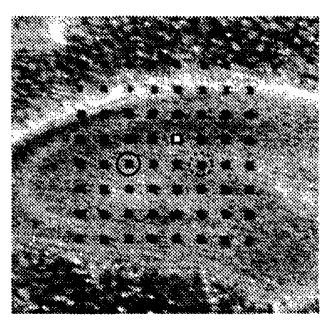

C

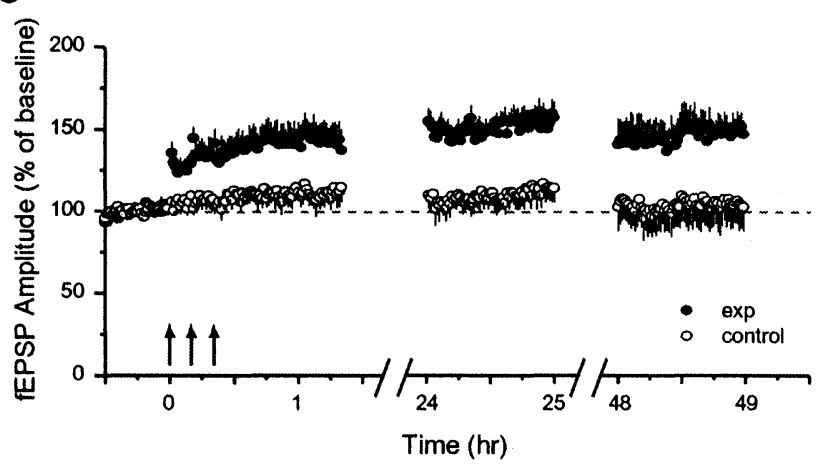

B
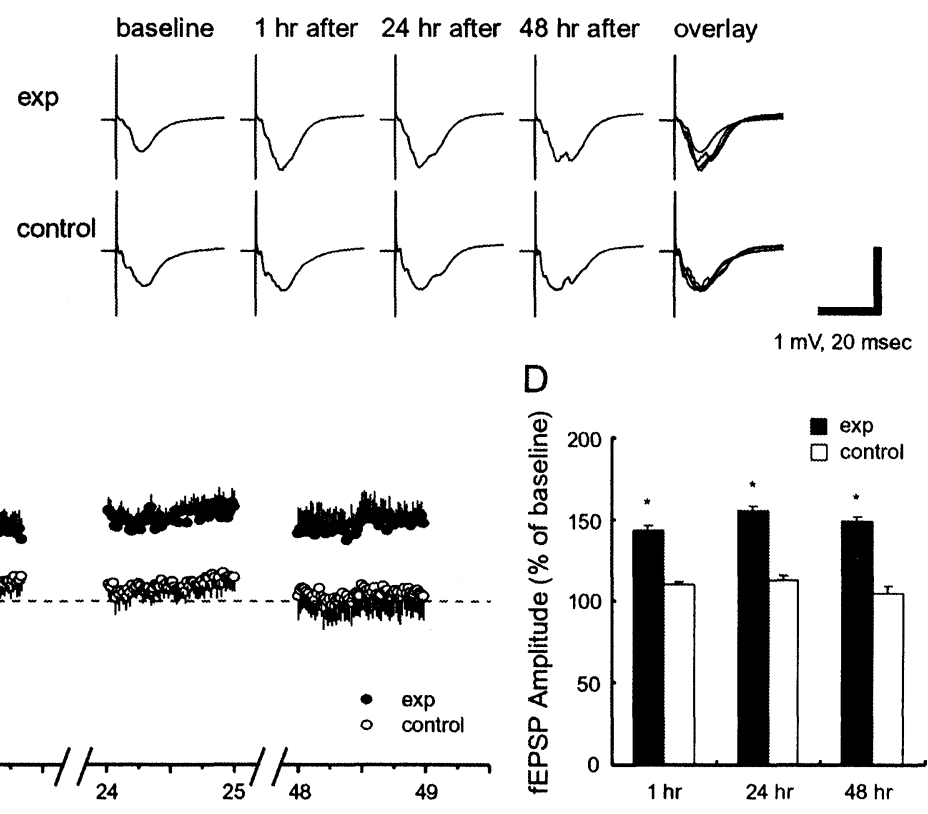

Fig. 1: Long lasting recording of long-term potentiation in cultured hippocampal slices. (A) Micrograph of a hippocampal slice cultured on an MED probe. Inter-electrode distance is $150 \mu \mathrm{m}$. The recording electrode is indicated by a white square, while stimulation electrodes are indicated by a solid circle (tetanized pathway (exp)) and a dotted circle (control pathway). (B) fEPSPs evoked by Schaffer fiber were recorded in field CA1 before and after tetanus stimulation. fEPSPs evoked by stimulation of the tetanized pathway (exp) are shown on the top row and fEPSPs evoked by stimulation of the control pathway (control) are shown on the bottom row (each response is the average of 10 sweeps; typical examples are shown). (C) Summarized graph of long lasting LTP recording. The maximum amplitude of fEPSP was determined and calculated as percentage of averaged baseline values (means \pm S.E.M., $n=8$ ). Arrows indicate time of high frequency stimulation. (D) LTP amplitude was averaged over a $10 \min$ period at $1 \mathrm{~h}, 24 \mathrm{~h}$ and $48 \mathrm{~h}$ after high frequency stimulation, and was expressed as percentage of baseline values. 


\section{Effect of APV on LTP induction in cultured hippocampal slices}

To determine whether the long-lasting LTP induced in slices cultured on MED probes was similar to the LTP typically induced in acute or cultured hippocampal slices, we applied tetanus stimulation in the presence of the NMDA receptor antagonist, D-APV. When APV $(50 \mu \mathrm{M})$ was applied $30 \mathrm{~min}$ before and during high frequency stimulation, no LTP was observed (relative amplitudes of test and control pathways were $113.9 \pm 0.9 \%$ and $112.2 \pm 1.0 \% 1 \mathrm{~h}$ after tetanus and $102.5 \pm 2.0 \%$ and $102.2 \pm 1.4 \% 3 \mathrm{~h}$ after APV washout; $\mathrm{n}=11$ ).

On the other hand, when the high-frequency stimulation was delivered $3 \mathrm{~h}$ after APV washout, LTP was again elicited and to the same degree as that in naïve slices $(146.5 \pm 2.8 \%$ and $116.4 \pm 2.8 \%$ in test and control pathways, respectively; $n=5$; data not shown). Such potentiation also lasted more than $1 \mathrm{~d}$. To further assess the effects of APV on synaptic plasticity, we determined the synaptic responses $1 \mathrm{~d}$ after high-frequency stimulation in the presence of APV and after APV washout. The synaptic responses remained unmodified when compared with their baseline values, regardless of whether the slices had been treated with APV and whether they had been tetanized in the presence of APV. This result clearly indicates that, under our conditions, high-frequency stimulation did not produce an NMDA receptor-independent form of LTP.

To eliminate the possibility that the lack of modification of synaptic responses was due to some deleterious effects of tetanization in the presence of APV, we tested the effects of highfrequency stimulation $1 \mathrm{~d}$ after APV washout. LTP was elicited in 6 of 8 slices, and the magnitude of LTP was similar to that produced in naïve slices (Fig. 2) (average relative amplitudes expressed as percent of initial baselines were $153.1 \pm 2.5 \%$ and $116.2 \pm 1.7 \%$ after $1 \mathrm{~h}$ in the test and control pathways, respectively; means \pm S.E.M. of 6 experiments).

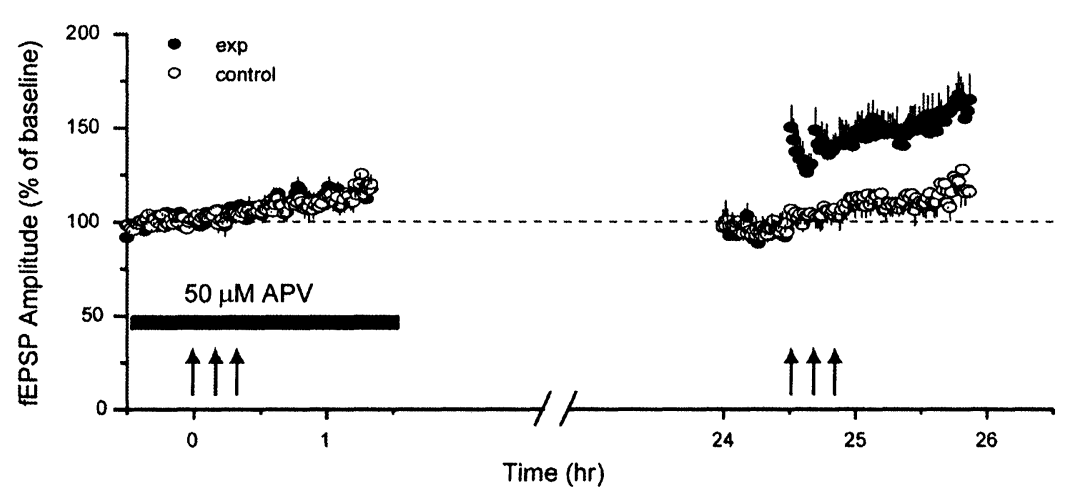

Fig. 2: Reversible blockade of LTP by APV in cultured hippocampal slices. High frequency stimulation was applied to one pathway (exp) while another unstimulated pathway received low frequency stimulation (control) in the presence of APV $(50 \mu \mathrm{M})$. Slices were returned to the incubator in culture medium without APV, and were tested the following day. Another high frequency stimulation was applied to the same pathway and responses measured for another $2 \mathrm{~h}$. Each point represents the mean \pm S.E.M. $(n=6)$ of the relative amplitude of fEPSPS in both pathways. Arrows indicate time of high frequency stimulation. 


\section{DISCUSSION}

The results of the present study indicate that trains of high-frequency stimulation in cultured hippocampal slices elicit a long lasting form of LTP that, in a significant number of slices, can be recorded for several days. This form of LTP was completely blocked by APV, an antagonist of NMDA receptors. The blockade was completely reversible as LTP could still be induced $3 \mathrm{~h}$ or $24 \mathrm{~h}$ after APV washout. Thus, this form of LTP exhibits features similar to those of the typical LTP elicited in CA1 of acute or cultured hippocampal slices. In this regard, our data regarding the probability and the amplitude of LTP in hippocampal slices cultured for $10 \mathrm{~d}$ agree with prior results of Muller et al. (1993).

Our results also indicate that some LTP in field CA1 of a subset of cultured hippocampal slices can occur without showing any significant decay over more than $2 \mathrm{~d}$, a result reminiscent of what has been reported for in vivo LTP in field CA1 (Staubli \& Lynch, 1987). To our knowledge, this is the first report indicating that an in vitro preparation provides duration for LTP similar to that found in vivo. This finding indicates that this experimental model might be ideally suited to study mechanisms underlying LTP maintenance and consolidation. Furthermore, as cultured slices have been successfully used with various mutant mice, our results open the way for a wide range of studies related to the long-term recording of synaptic modifications resulting from a broad spectrum of manipulations.

\section{REFERENCES}

Benke TA, Luthi A, Isaac JTR, Collingridge GL. 1998. Modulation of AMPA receptor unitary conductance by synaptic activity. Nature 393: 793-797.

Chang FL, Greennough WT. 1984. Transient and enduring morphological correlates of synaptic activity and efficacy change in the rat hippocampal slice. Brain Res 309: 35-46.

Desmond NL, Levy WB. 1986. Changes in the postsynaptic density with long-term potentiation in the dentate gyrus. Brain Res 253: 476-482.

Frey U, Morris RGM. 1997. Synaptic tagging and long-term potentiation. Science 385: 533-536.

Geinisman Y, Detoledo-Morrell L, Morrell F. 1991. Induction of long-term potentiation is associated with an increase in the number of axospinous synapses with segmented postsynaptic densities. Brain Res 566: 77-88.

Lee L, Oliver M, Schottler F, Lynch G. 1981. Electron microscopic studies of brain slices: The effects of high-frequency stimulation on dendritic ultrastructure. In: Kerkut GA, Wheal HV, eds, Electrophsiology of isolated mammalian CNS preparations. New York, NY, USA: Academic Press; 189-211.

Luscher C, Nicoll RA, Malenka RC, Muller D. 2000. Synaptic plasticity and dynamic modulation of the postsynaptic membrane. Nat Neurosci 3: 545-550.

Lynch G, Baudry M. 1991. Reevaluating the constraints on hypotheses regarding LTP expression. Hippocampus 1: 9-14.

Malenka R, Nicoll R. 1999. Long-term potentiation-a decade of progress? Science 285: 1870-1874.

Malinow R, Malenka RC. 2002. AMPA receptor trafficking and synaptic plasticity. Ann Rev Neurosci 25: 103-126.

Muller D, Buchs PA, Stoppini L. 1993. Time course of synaptic development in hippocampal organotypic cultures. Dev Brain Res 71: 93-100.

Shi SH, Hayashi Y, Petralia RS, Zaman SH, Wenthold RJ, Svoboda K, et al. 1999. Rapid spine delivery and redistribution of AMPA receptors after synaptic NMDA receptor activation. Science 284: $1811-$ 1816.

Shimono K, Baudry M, Panchenko V, Taketani M. 2002. Chronic multichannel recording from organotypic hippocampal slice cultures: protection from excitotoxic effects of NMDA by noncompetitive NMDA antagonists. J Neurosci Methods 120: 193-202.

Staubli U, Lynch G. 1987. Stable hippocampal longterm potentiation elicited by 'theta' pattern stimulation. Brain Res 435: 227-234.

Toni N, Buchs PA, Nikonenko I, Bron CR, Muller D. 1999. LTP promotes formation of multiple spine synapses between a single axon terminal and a dendrite. Nature 402: 421-425. 

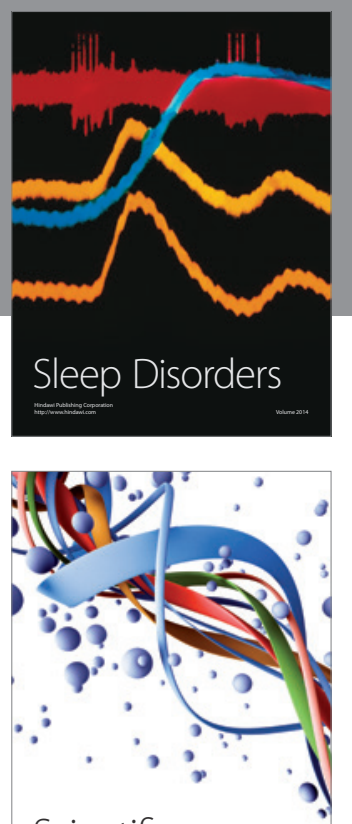

Scientifica
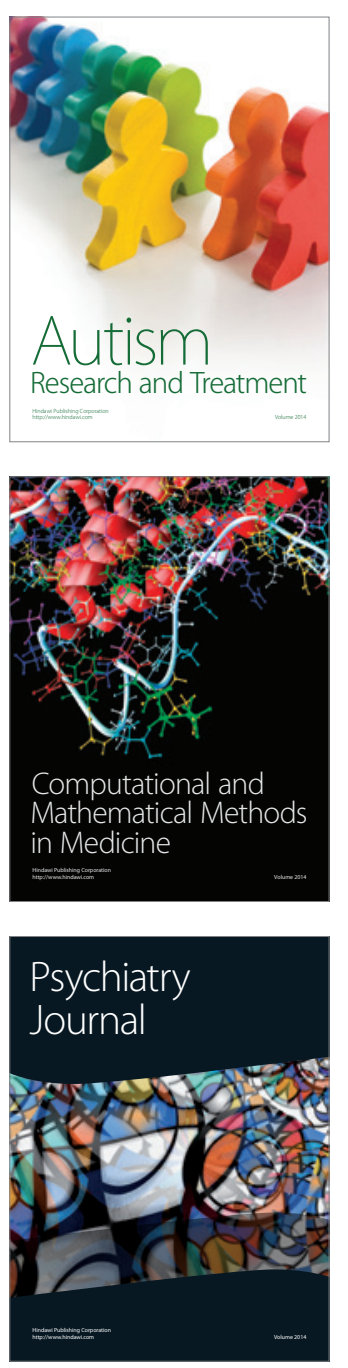
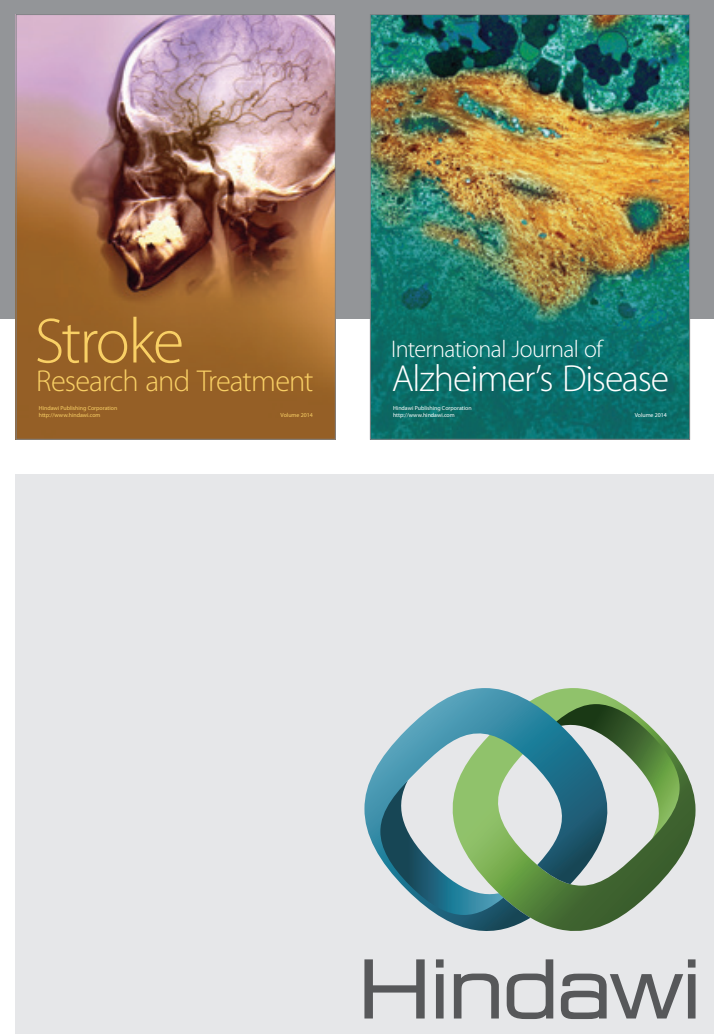

Submit your manuscripts at

http://www.hindawi.com
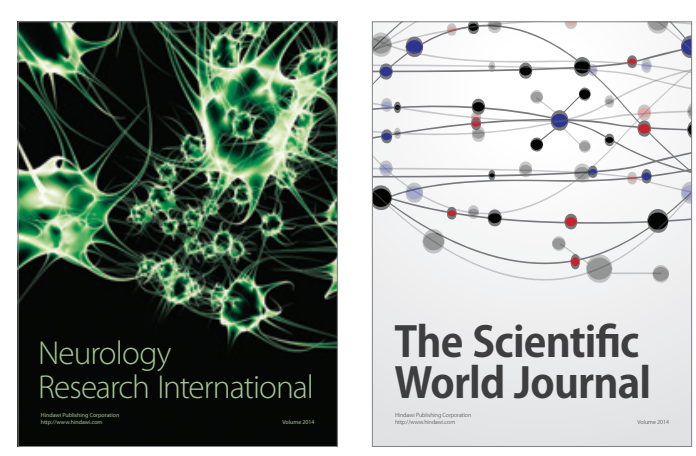

The Scientific World Journal

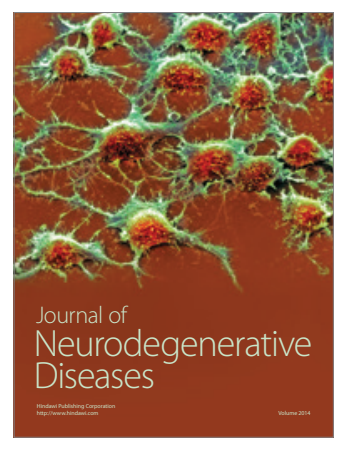

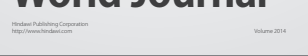

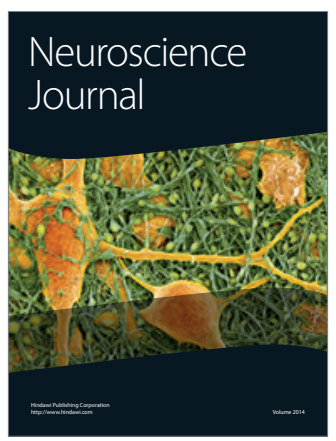

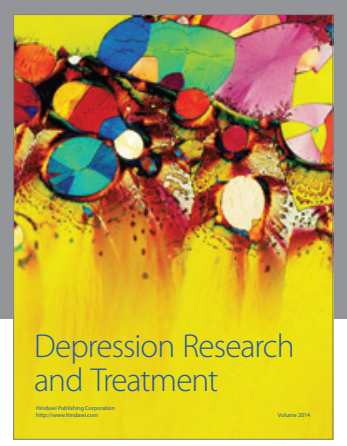
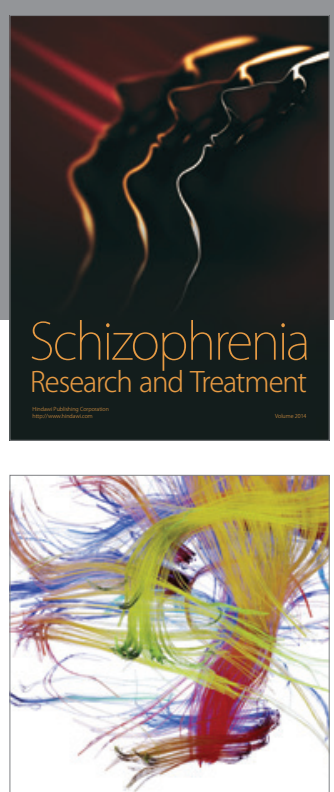

Brain Science

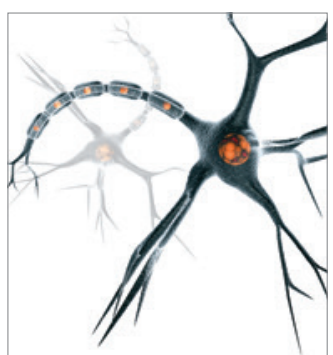

Neural Plasticity
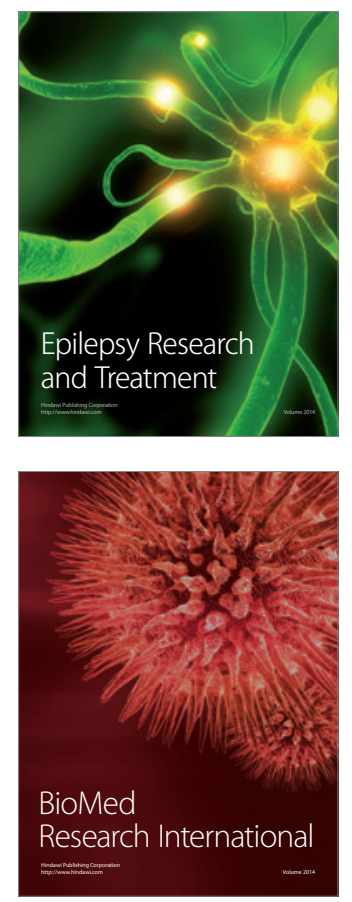

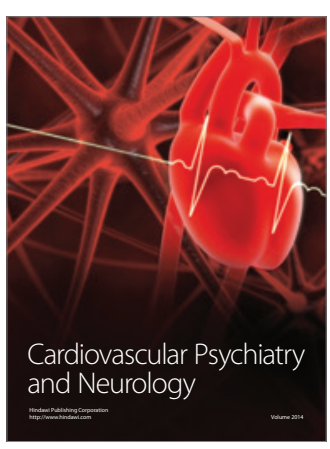

Parkinson's

Disease
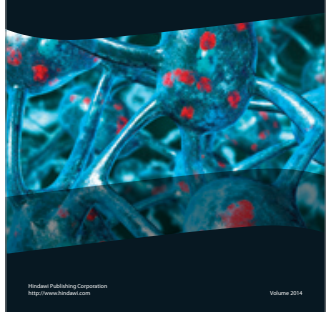